\title{
ATP Production and Necrosis Formation in a Tumour Spheroid Model
}

\author{
A. Bertuzzi ${ }^{a}$, A. Fasano $^{b}$, A. Gandolfi ${ }^{a 1}$ and C. Sinisgalli ${ }^{a}$ \\ ${ }^{a}$ Istituto di Analisi dei Sistemi ed Informatica "A. Ruberti" - CNR \\ Viale Manzoni 30, 00185 Roma, Italy \\ ${ }^{b}$ Dipartimento di Matematica "U. Dini”, Università di Firenze \\ Viale Morgagni 67/A, 50134 Firenze, Italy
}

\begin{abstract}
Mathematical models of tumour spheroids, proposed since the early seventies, have been generally formulated in terms of a single diffusive nutrient which is critical for cell replication and cell viability. Only recently, attempts have been made to incorporate in the models the cell energy metabolism, by considering the interplay between glucose, oxygen and lactate (or $\mathrm{pH}$ ). By assuming glucose and lactate as the only fuel substrates, we propose a simple model for the cell ATP production which takes into account the main reactions that occur in the glycolytic and the oxidative pathway. Under the assumption that cell death occurs when ATP production falls below a critical level, we have studied the free boundary problem for the concentration of glucose, lactate and oxygen inside the spheroid viable rim. We show that the existence of a necrotic core is guaranteed for a sufficiently large size of the spheroid.
\end{abstract}

Key words: tumour spheroid, energy metabolism, diffusion, free boundary problems AMS subject classification: 35R35, 92C37, 92C50

\section{Introduction}

Multicellular tumour spheroids growing in vitro have been extensively investigated as experimental models of avascular tumour nodules [17]. During the growth of the spheroid the fraction of proliferating cells decreases, and when the cells become deprived of oxygen, glucose and other nutrients, and/or metabolic waste accumulates, cell death occurs. Thus, in an advanced stage of

\footnotetext{
${ }^{1}$ Corresponding author. E-mail: gandolfi@iasi.cnr.it
} 
growth, the spheroid shows an outer viable rim (whose thickness ranges from about $100 \mu \mathrm{m}$ to $250 \mu \mathrm{m}$ ) surrounding a necrotic core. Because of the degradation of dead cells in the necrotic core, the spheroid growth eventually saturates with a final diameter of $1-3 \mathrm{~mm}$.

Many mathematical models have been proposed to describe the spheroid growth by using either a continuum or a discrete approach (see [2] for a comprehensive review). In almost all of these models, cell proliferation and cell death are assumed to depend on the concentration of a single critical chemical, diffusing from the external medium into the spheroid mass. The diffusion of glucose, oxygen and other chemicals has been included in a spheroid model proposed in [6]. In that model, cell proliferation rate was taken to be an empirical function of glucose, oxygen and hydrogen ion concentrations. Both glucose and oxygen transport were included in a discrete lattice model of the spheroid growth [13]. More recently, the interplay among oxygen and other substrates (such as glucose and lactate) in the cell energy metabolism, i.e. in the intracellular ATP production, has been incorporated in a continuous spheroid model [18] and in a cellular automata tumour growth model [7].

In the present paper, by assuming glucose and lactate as the only fuel substrates, we propose a simple model for the cell ATP production which takes into account the main reactions that occur in the glycolytic and the oxidative pathway. Under the assumption that cell death occurs when ATP production falls below a critical level, we have studied the free boundary problem for the concentration of glucose, lactate and oxygen inside the spheroid viable rim. We show that the existence of a necrotic core is guaranteed for spheroids of a sufficiently large size.

\section{Cell energy metabolism}

\subsection{Description of main biochemical reactions}

Cell energy metabolism in vivo involves glucose, fatty acids and amino acids. As we will focus here on in vitro multicellular tumour spheroids, we will only consider the three key metabolites glucose, lactate and oxygen. The basic biochemical reactions are depicted in Fig. 1 (see e.g. [16] for a detailed description).

The first step of glucose metabolism is glycolysis, in which a sequence of enzymatic reactions converts glucose into pyruvate with the simultaneous net production of two ATP molecules ${ }^{1}$ and the conversion of two $\mathrm{NAD}^{+}$into NADH molecules:

$$
\text { Glucose }+2 \mathrm{NAD}^{+}+2 \mathrm{P}_{\mathrm{i}}+2 \mathrm{ADP} \rightarrow 2 \text { Pyruvate }+2\left(\mathrm{NADH}+\mathrm{H}^{+}\right)+2 \mathrm{ATP}+2 \mathrm{H}_{2} \mathrm{O} \text {. }
$$

As shown by the scheme of Fig. 1, pyruvate may either be reversibly converted into lactate or undergo complete oxidation in the Krebs cycle and the associated electron transport system (ETS). The former reaction is given by

$$
\text { Pyruvate }+\mathrm{NADH}+\mathrm{H}^{+} \rightleftharpoons \text { Lactate }+\mathrm{NAD}^{+} .
$$

\footnotetext{
${ }^{1}$ Abbreviations: ATP, adenosine triphosphate; ADP, adenosine diphosphate; $\mathrm{P}_{\mathrm{i}}$, inorganic orthophosphate; GTP, guanosine triphosphate; GDP, guanosine diphosphate; $\mathrm{NAD}^{+}$, nicotinamide adenine dinucleotide (oxidized form); $\mathrm{NADH}$, nicotinamide adenine dinucleotide (reduced form); FAD, flavin adenine dinucleotide (oxidized form); $\mathrm{FADH}_{2}$, flavin adenine dinucleotide (reduced form); Ac-CoA, acetyl-CoA.
} 


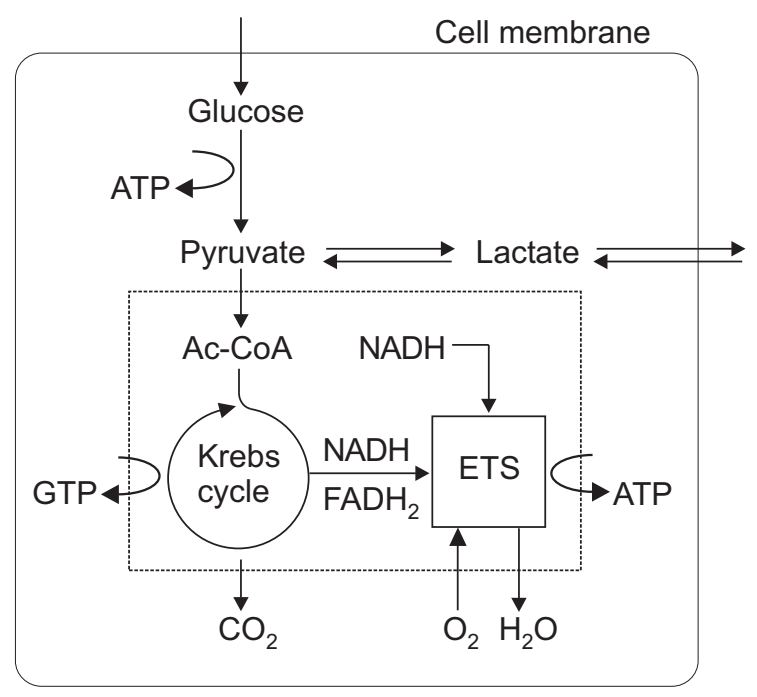

Figure 1: Simplified scheme of glucose metabolism. The inner box represents the mitochondria.

Pyruvate oxidation occurs through multiple steps, but the net reaction may be written as follows:

$$
\begin{aligned}
& \text { Pyruvate }+4 \mathrm{NAD}^{+}+\mathrm{FAD}+3 \mathrm{H}_{2} \mathrm{O}+\mathrm{P}_{\mathrm{i}}+\mathrm{GDP} \\
& \rightarrow 3 \mathrm{CO}_{2}+4\left(\mathrm{NADH}+\mathrm{H}^{+}\right)+\mathrm{FADH}_{2}+\mathrm{GTP} .
\end{aligned}
$$

The NADH produced in the pyruvate $\rightarrow$ acetyl-CoA step, in the Krebs cycle, and in the glycolytic step, together with the $\mathrm{FADH}_{2}$ produced in the Krebs cycle, are oxidized in the so-called electron transport system:

$$
10\left(\mathrm{NADH}+\mathrm{H}^{+}\right)+2 \mathrm{FADH}_{2}+6 \mathrm{O}_{2} \rightarrow 10 \mathrm{NAD}^{+}+2 \mathrm{FAD}+12 \mathrm{H}_{2} \mathrm{O} .
$$

In the above reaction, that refers to the oxidation of 1 glucose molecule, $2 \mathrm{NADH}$ molecules are produced in the pyruvate $\rightarrow$ acetyl-CoA step, 6 NADH molecules are generated in the Krebs cycle and the remaining 2 molecules come from the glycolytic step. We note that this reaction provides all the $\mathrm{NAD}^{+}$and FAD molecules required. The reactions in the electron transport system yield the energy necessary for the phosphorylation of ADP into ATP. The efficiency of this process appears to be variable depending on cell type and cell condition. The transformation of 1 NADH molecule in $\mathrm{NAD}^{+}$provides energy for the formation, in average, of about 2.5 molecules of ATP, whereas 1 $\mathrm{FADH}_{2}$ generates about 1.5 ATP molecules [12]. Also, we remark that the GTP molecule produced in the Krebs cycle is equivalent to an ATP molecule.

Let us consider a cell at the stationary state, that is when the intracellular concentrations are constant in time. Under the hypothesis that glucose, lactate and oxygen are the only metabolites that can be exchanged with extracellular space, the reactions (2.1)-(2.4) impose a relationship among the consumption rates $\left(\mathrm{mol} \cdot \mathrm{cell}^{-1} \cdot \mathrm{s}^{-1}\right)$ of these compounds. We have (see the Appendix)

$$
f_{O}=6 f_{G}+3 f_{L},
$$


where $f_{O}>0$ and $f_{G}>0$ are the consumption rates of $\mathrm{O}_{2}$ and glucose, respectively, and $f_{L}$ is the consumption rate of lactate. Note that $f_{L}$ can also be negative because, according to the reaction (2.2), there can be a net production of lactate. The production rate of ATP can be expressed in terms of the above rates. Denoting by $\eta_{1}$ and $\eta_{2}$ the efficiency of the phosphorylation of ADP into ATP related to the oxidation of $\mathrm{NADH}$ and $\mathrm{FADH}_{2}$, respectively, we obtain from reactions (2.1)-(2.4) (see the Appendix)

$$
f_{A T P}=2 f_{G}+\left(5 \eta_{1}+\eta_{2}+1\right) f_{O} / 3 .
$$

Equivalent expressions, that include $f_{L}$, may be derived by means of (2.5).

\subsection{A simple mathematical model}

Equations (2.5)-(2.6) give relations among metabolite fluxes that are implied by the stoichiometry of reactions, but say nothing on the extent of these fluxes. For our aims, moreover, it is necessary to express the consumption/production rates in terms of the extracellular concentrations. Let us consider a cell surrounded by a medium in which glucose, oxygen and lactate have constant concentrations $\sigma_{G}, \sigma_{O}$ and, respectively, $\sigma_{L}$. The respective intracellular concentrations will be denoted as $\bar{\sigma}_{G}, \bar{\sigma}_{O}$, and $\bar{\sigma}_{L}$.

We assume: (i) glucose uptake follows a Michaelis-Menten kinetics and all glucose taken up by cells is consumed $[6,18]$; (ii) the possible exchange of pyruvate with the extracellular medium is disregarded; (iii) transmembrane transport of lactate has a saturable component dependent only on extracellular lactate concentration, plus a nonsaturable purely passive component $[8,3]$; (iv) because of the high permeability of cell membrane to oxygen [1], oxygen concentration inside the cell is equal to the extracellular concentration; $(v)$ the pyruvate flow toward the Krebs cycle is proportional to the product of pyruvate and oxygen concentrations.

On the basis of the above assumptions, the rate of glucose uptake is given by

$$
f_{G}\left(\sigma_{G}\right)=F_{G} \frac{\sigma_{G}}{K_{G}+\sigma_{G}}
$$

where $F_{G}$ is the maximal rate of uptake and $K_{G}$ is the Michaelis-Menten constant. For the intracellular concentrations of pyruvate and lactate we write the following equations:

$$
\begin{aligned}
v \frac{d \bar{\sigma}_{P}}{d t} & =2 f_{G}\left(\sigma_{G}\right)-\psi\left(\bar{\sigma}_{P}, \bar{\sigma}_{L}\right)-\phi_{P}\left(\bar{\sigma}_{P}, \sigma_{O}\right), \\
v \frac{d \bar{\sigma}_{L}}{d t} & =\psi\left(\bar{\sigma}_{P}, \bar{\sigma}_{L}\right)+F_{L} \frac{\sigma_{L}}{K_{L}+\sigma_{L}}+v \bar{h}\left(\sigma_{L}-\bar{\sigma}_{L}\right),
\end{aligned}
$$

where $v$ is the cell volume, $\psi$ is the net flow of pyruvate to lactate, $\phi_{P}$ is the pyruvate flow toward the Krebs cycle, $F_{L}$ and $K_{L}$ are the parameters characterizing the saturable component of lactate transmembrane transport, and $\bar{h}$ is the transfer coefficient of the diffusive transport. Although the reversible reaction between pyruvate and lactate is an enzymatic reaction, we simply represent it by a first-order kinetics, writing

$$
\psi\left(\bar{\sigma}_{P}, \bar{\sigma}_{L}\right)=v\left(k_{+} \bar{\sigma}_{P}-k_{-} \bar{\sigma}_{L}\right),
$$


where $k_{+}$and $k_{-}$are the forward and, respectively, the backward rate constants. The complex reactions in the Krebs cycle and the electron transport system that lead to pyruvate oxidation, will be summarized in the simplest way according to assumption $(v)$ :

$$
\phi_{P}\left(\bar{\sigma}_{P}, \sigma_{O}\right)=v \gamma \bar{\sigma}_{P} \sigma_{O}
$$

At the steady state, from Eqs. (2.7)-(2.11), the intracellular concentrations of pyruvate and lactate can be derived in terms of the extracellular concentrations:

$$
\begin{aligned}
\bar{\sigma}_{P} & =\frac{1}{k_{+} \alpha+\gamma \sigma_{O}}\left[2 \frac{F_{G}}{v} \frac{\sigma_{G}}{K_{G}+\sigma_{G}}+(1-\alpha) \frac{F_{L}}{v} \frac{\sigma_{L}}{K_{L}+\sigma_{L}}+k_{-} \alpha \sigma_{L}\right], \\
\bar{\sigma}_{L} & =\frac{1}{k_{-}+\bar{h}} \frac{1}{k_{+} \alpha+\gamma \sigma_{O}}\left[2 k_{+} \frac{F_{G}}{v} \frac{\sigma_{G}}{K_{G}+\sigma_{G}}+\left(k_{+}+\gamma \sigma_{O}\right)\left(\frac{F_{L}}{v} \frac{\sigma_{L}}{K_{L}+\sigma_{L}}+\bar{h} \sigma_{L}\right)\right],
\end{aligned}
$$

where $\alpha=\bar{h} /\left(k_{-}+\bar{h}\right)$. As seen in the Appendix, from the stoichiometry of the reactions at the steady state we have

$$
f_{O}=3 \phi_{P}
$$

Therefore we can write:

$$
f_{O}\left(\sigma_{G}, \sigma_{L}, \sigma_{O}\right)=\left(6 F_{G} \frac{\sigma_{G}}{K_{G}+\sigma_{G}}+a_{1} \frac{\sigma_{L}}{K_{L}+\sigma_{L}}+a_{2} \sigma_{L}\right) \frac{\sigma_{O}}{K_{O}+\sigma_{O}}
$$

where

$$
a_{1}=3 F_{L} \frac{k_{-}}{k_{-}+\bar{h}}, \quad a_{2}=3 \frac{k_{-} \bar{h}}{k_{-}+\bar{h}} v, \quad K_{O}=\frac{k_{+} \bar{h}}{\gamma\left(k_{-}+\bar{h}\right)} .
$$

We note that $f_{O}$ is a Michaelis-Menten function of $\sigma_{O}$, with the maximal consumption rate that depends on the extracellular concentrations of glucose and lactate. For $f_{L}$, by observing that $f_{L}=F_{L} \sigma_{L} /\left(K_{L}+\sigma_{L}\right)+v \bar{h}\left(\sigma_{L}-\bar{\sigma}_{L}\right)$, we obtain:

$$
f_{L}\left(\sigma_{G}, \sigma_{L}, \sigma_{O}\right)=\frac{1}{3\left(K_{O}+\sigma_{O}\right)}\left[\left(a_{1} \frac{\sigma_{L}}{K_{L}+\sigma_{L}}+a_{2} \sigma_{L}\right) \sigma_{O}-6 F_{G} K_{O} \frac{\sigma_{G}}{K_{G}+\sigma_{G}}\right] .
$$

Equation (2.13) shows that $f_{L}$ may be positive or negative according to the value of oxygen concentration: when $\sigma_{O} \rightarrow 0$, we will have $f_{L}<0$, that is, lactate production. Note that $f_{G}, f_{L}$ and $f_{O}$ as given by (2.7), (2.13) and (2.12) respectively, satisfy the relationship (2.5). We also remark that, although the model depends on eight physical parameters, the consumption rates only depend on the six parameter combinations: $F_{G}, K_{G}, a_{1}, a_{2}, K_{L}$, and $K_{O}$.

Remark 2..1. Experimental evidences have suggested that energy metabolism of tumour cells mainly relies on the glycolytic pathway for ATP production, thus exhibiting enhanced lactate production and the formation of an acidic environment. These observations led O. Warburg to the notion of the glycolytic phenotype of cancer cells [19]. Recently, however, this view has been questioned by stressing that cells may rely on glycolysis because of the prevailing hypoxic conditions in tumours [11]. In our model, the balance between the glycolytic and the aerobic ATP production is governed by the parameters $k_{+}$and $\gamma$. From Eqs. (2.6) and (2.12), we see that if the ratio $k_{+} / \gamma$ is large, the aerobic production of ATP is small even in the presence of high oxygen concentrations, and thus the cells would express a glycolytic phenotype. 
Remark 2..2. It can be useful to compare the above model with an energy metabolism model recently proposed [18]. In that paper, the glucose consumption rate has the same expression as in Eq. (2.7), whereas the rates of lactate and oxygen consumption are assumed to be smaller or equal to a Michaelis-Menten function:

$$
f_{L} \leq F_{L} \frac{\sigma_{L}}{K_{L}+\sigma_{L}}, \quad f_{O} \leq F_{O} \frac{\sigma_{O}}{K_{O}+\sigma_{O}} .
$$

The setting of [18] is characterized by the presence of two regimens, that satisfy relationship (2.5). If oxygen concentration is large enough so that

$$
F_{O} \frac{\sigma_{O}}{K_{O}+\sigma_{O}} \geq 6 F_{G} \frac{\sigma_{G}}{K_{G}+\sigma_{G}}+3 F_{L} \frac{\sigma_{L}}{K_{L}+\sigma_{L}},
$$

then in [18] it is assumed that lactate is consumed at the maximal rate,

$$
f_{L}=F_{L} \frac{\sigma_{L}}{K_{L}+\sigma_{L}}
$$

so that from Eq. (2.5)

$$
f_{O}=6 F_{G} \frac{\sigma_{G}}{K_{G}+\sigma_{G}}+3 F_{L} \frac{\sigma_{L}}{K_{L}+\sigma_{L}} .
$$

Otherwise (hypoxic regimen), it is assumed that oxygen is consumed at the maximal rate,

$$
f_{O}=F_{O} \frac{\sigma_{O}}{K_{O}+\sigma_{O}}
$$

and then

$$
f_{L}=-2 F_{G} \frac{\sigma_{G}}{K_{G}+\sigma_{G}}+\frac{1}{3} F_{O} \frac{\sigma_{O}}{K_{O}+\sigma_{O}} .
$$

We note that, in the normoxic condition, the model in [18] supposes the pyruvate $\rightleftharpoons$ lactate reaction to completely favour the formation of pyruvate. In fact, if $\sigma_{L}=0$ the model predicts $f_{L}=0$, whereas if the reaction has a finite equilibrium, some lactate production would occur. Moreover, in the hypoxic condition, lactate production is independent of the extracellular lactate concentration. Figure 2 exemplifies the behaviour of $f_{O}$ and $f_{L}$ according to our model and to the model proposed in [18].

Remark 2..3. Experimental data $[9,6,14]$ have shown that glucose consumption increases when oxygen concentration decreases (the so-called Pasteur effect) and that, at physiological $\mathrm{pH}$, oxygen consumption increases when glucose concentration decreases (the so-called Crabtree effect). Both the model proposed in [18] and our model, neglect these effects. A mechanistic representation of these phenomena appears rather complex. Expressions for the glucose and oxygen consumption including the above effects were proposed on an empirical basis [6], and have been used in a recent model of tumour growth incorporating cell metabolism [7]. These effects appear to be dependent on the $\mathrm{pH}$ of cell environment (for instance, the Crabtree effect disappears in an acidic environment). We observe that, although the production of $\mathrm{H}^{+}$ions is related to lactic acid production, the description of $\mathrm{pH}$ changes is complicated by the presence of an intracellular buffer (see the mathematical model in [5] and, for a simplified approach, [7]). The importance of the $\mathrm{pH}$ of microenvironment in connection with tumour invasion has been pointed out in $[10,15]$. 

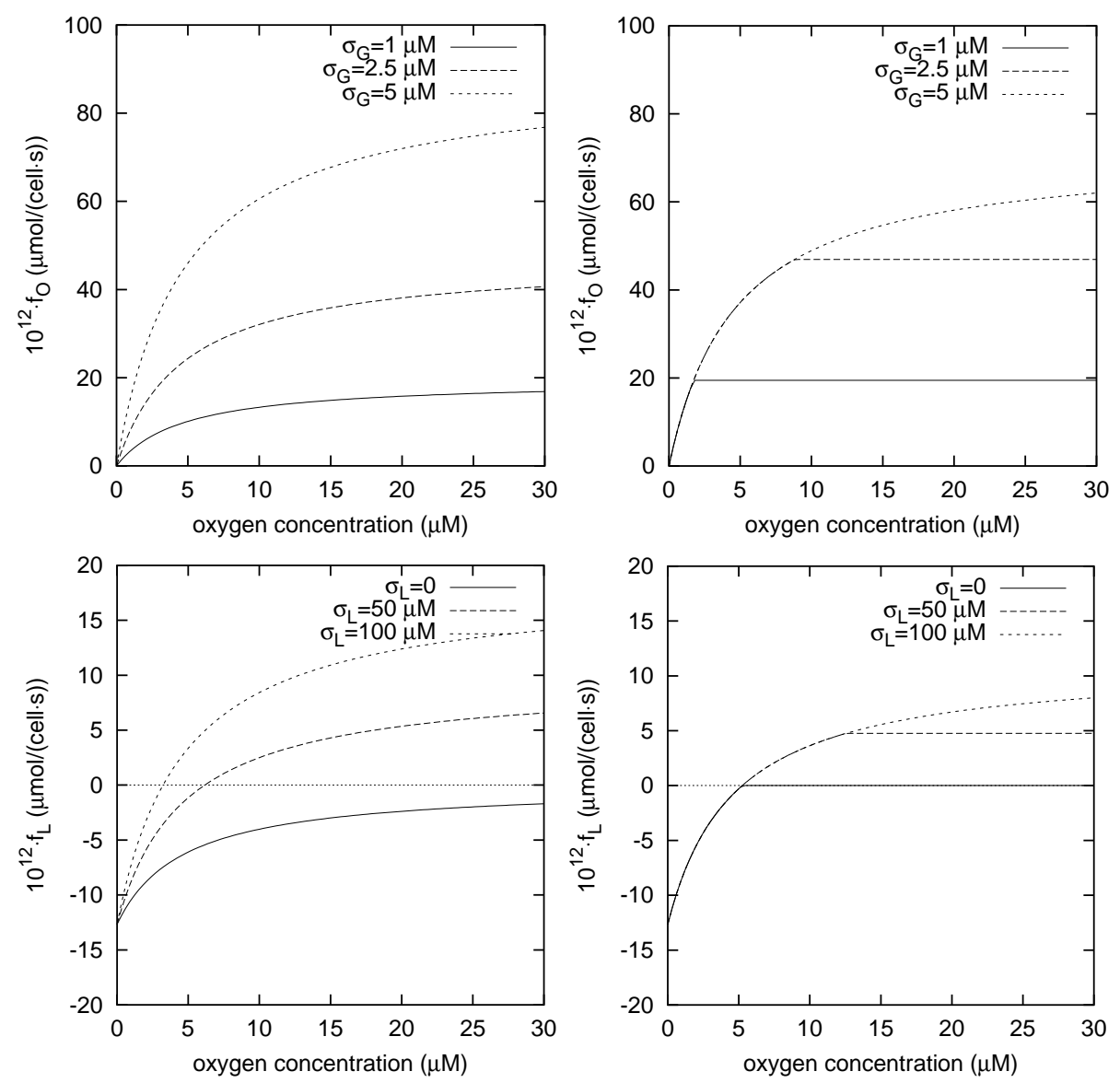

Figure 2: Upper panels: Oxygen consumption rate when $\sigma_{L}=0$ according to Eq. (2.12) (left) and according to model in [18] (right). Lower panels: Lactate consumption rate when $\sigma_{G}=2 \mu \mathrm{M}$ according to Eq. (2.13) (left) and according to model in [18] (right). Parameter values (concentrations in $\mu \mathrm{M}$, consumption rates $\mu \mathrm{mol} /(\mathrm{cell} \cdot \mathrm{s})$ ): $F_{G}=1.33 \cdot 10^{-10}, K_{G}=40.0, F_{O}=7.16 \cdot 10^{-11}$, $K_{O}=4.64[18], F_{L}=1 \cdot 10^{-10}, K_{L}=1000, a_{1}=6 \cdot 10^{-12}, a_{2}=7.8 \cdot 10^{-16}$.

\section{Necrotic core formation in multicellular spheroids}

We consider a spheroid of radius $R$, in which the nutrient consumption rates of all viable cells depend on the concentrations of the chemicals according to the expressions given in the previous section. Since the growth dynamics of the spheroid is much slower than the process of diffusion of the nutrients, we will consider the diffusion process (in spherical symmetry) to be at the quasi-steady state at any time. The extracellular concentrations $\sigma_{G}, \sigma_{L}, \sigma_{O}$ are prescribed at the boundary:

$$
\sigma_{i}(R)=\sigma_{i}^{*}>0, \quad i=G, O, \quad \sigma_{L}(R)=\sigma_{L}^{*} \geq 0 .
$$

Of course all the derivatives $\sigma_{i}^{\prime}$ vanish for $r=0$ by symmetry (whether or not a necrotic core is present). 
We take the rate of ATP production as a viability index for the cells, and we assume that cells die when $f_{A T P}$, as given by Eq. (2.6), falls to a threshold $f_{N}$. According to this choice, the viable rim of the spheroid is the region $\rho_{N}<r<R$ defined by the inequality

$$
f_{A T P}(r)>f_{N}
$$

We obviously assume that the concentrations at the boundary $R$ are such that $f_{A T P}(R)>f_{N}$. Of course $\rho_{N}$ can be zero (no necrotic core). In the viable region the concentrations $\sigma_{G}, \sigma_{L}, \sigma_{O}$ obey the equations

$$
\begin{aligned}
\nu_{E} D_{G} \Delta \sigma_{G} & =\left(1-\nu_{E}\right) f_{G}\left(\sigma_{G}\right), \\
\nu_{E} D_{L} \Delta \sigma_{L} & =\left(1-\nu_{E}\right) f_{L}\left(\sigma_{G}, \sigma_{L}, \sigma_{O}\right), \\
D_{O} \Delta \sigma_{O} & =\left(1-\nu_{E}\right) f_{O}\left(\sigma_{G}, \sigma_{L}, \sigma_{O}\right),
\end{aligned}
$$

where $\nu_{E}$ is the volume fraction of extracellular liquid, supposed constant, and the symbols $D_{i}$ denote the diffusivities. Since glucose is only taken up by cells, its transport consists in a diffusion in the extracellular space. The same assumption is done for lactate transport, although in this case the compound can either enter or leave the cells: in this way we have neglected the possible contribution to lactate transport of diffusion in the intracellular space. As previously noted, oxygen permeates the tissue without appreciable jump across the cell membrane, so that its diffusion is considered to occur in the whole space. We note that the quantities $f_{i}, i=G, L, O$, in Eqs. (3.2)-(3.4) represent consumption/production rates per unit volume and thus they can be taken proportional to the rates per cell defined in the previous section by Eqs. (2.7), (2.13) and (2.12), respectively. However, for notational simplicity, we have used the same symbols. We remark that the values of diffusivities (typical values: $D_{G}=1.05 \times 10^{-6} \mathrm{~cm}^{2} / \mathrm{s}, D_{L}=1.78 \times 10^{-6} \mathrm{~cm}^{2} / \mathrm{s}$, $D_{O}=1.82 \times 10^{-5} \mathrm{~cm}^{2} / \mathrm{s}$ [18]) guarantee that the contribution of the liquid flow to transport is negligible. In the following it is convenient to use the parameters defined as:

$$
\Gamma_{G}=\frac{1-\nu_{E}}{\nu_{E}} \frac{1}{D_{G}}, \quad \Gamma_{L}=\frac{1-\nu_{E}}{\nu_{E}} \frac{1}{D_{L}}, \quad \Gamma_{O}=\frac{1-\nu_{E}}{D_{O}} .
$$

When a necrotic core is present, i.e. $\rho_{N}>0$, the necrotic boundary bears the conditions

$$
\begin{aligned}
f_{A T P}\left(\rho_{N}\right) & =f_{N}, \\
\sigma_{i}^{\prime}\left(\rho_{N}\right) & =0, \quad i=G, L, O .
\end{aligned}
$$

In this section we start by proving the following

Proposition 3..1. For $R$ sufficiently large, $f_{A T P}(r)>f_{N}$ cannot hold in the whole interval $(0, R)$.

Let us first state a simple result.

Proposition 3..2. If the triple $\left(\sigma_{G}, \sigma_{L}, \sigma_{O}\right)$ is a solution of Eqs. (3.2)-(3.4) on (0, R), with boundary conditions (3.1) and no-flux conditions at $r=0$, none of these concentrations can vanish inside the spheroid. Moreover, $\sigma_{G}$ and $\sigma_{O}$ take their minimum for $r=0$. Finally, $\sigma_{G} \leq \sigma_{G}^{*}$, $\sigma_{O} \leq \sigma_{O}^{*}$ and $\sigma_{L}<\sigma_{L}^{*}+2\left(\Gamma_{L} / \Gamma_{G}\right) \sigma_{G}^{*}$. 
Proof. The positivity of $\sigma_{G}$ is immediate, since $\sigma_{G}$ cannot have a negative minimum (nor a positive maximum). Moreover, if for some $r^{\star} \in(0, R)$ it happens that $\sigma_{G}\left(r^{\star}\right)=0$, then necessarily $\sigma_{G}^{\prime}\left(r^{\star}\right)=0$ (otherwise a negative minimum would exist somewhere), but this is consistent with (3.2) only if $\sigma_{G} \equiv 0$, contradicting (3.1). Finally $\sigma_{G}$ cannot take a positive local minimum out of the center, because otherwise it would also take a local positive maximum in order to meet the condition $\sigma_{G}^{\prime}(0)=0$, which is excluded by the positivity of the r.h.s. of (3.2). Passing to the analysis of $\sigma_{L}, \sigma_{O}$, we provisionally replace $\sigma_{O}$ by its positive part $\sigma_{O}^{+}$in the expression of $f_{L}$. Having proved that $\sigma_{G}$, and hence $f_{G}$, is strictly positive, we see that if $\sigma_{L}$ is going to take a minimum it has to be positive (and $\sigma_{O}>0$ at the same time). Hence $\sigma_{L}>0$ in the whole sphere. Now we observe that the factor multiplying the ratio $\sigma_{O} /\left(K_{O}+\sigma_{O}\right)$ in $f_{O}$ is positive, and we may repeat the same argument used for $\sigma_{G}>0$ to conclude that $\sigma_{O}>0$ and takes its minimum for $r=0$. Therefore $\sigma_{O}^{+}=\sigma_{O}$ and the replacement we have made was just formal, so that our conclusions apply to the problem in its original form.

The inequalities $\sigma_{G} \leq \sigma_{G}^{*}, \sigma_{O} \leq \sigma_{O}^{*}$ are an immediate consequence of the fact that both the r.h.s. of (3.2) and (3.4) are positive. The bound on $\sigma_{L}$ follows by considering the combination $\tilde{\sigma}=2 \Gamma_{L} \sigma_{G}+\Gamma_{G} \sigma_{L}$, which satisfies the equation

$$
\Delta \tilde{\sigma}=2 \Gamma_{L} \Gamma_{G} f_{G} \frac{\sigma_{O}}{K_{O}+\sigma_{O}}>0
$$

and then it is bounded from above by its boundary value.

We can now prove Proposition 3..1.

Proof. of Proposition 3..1 Let $\left(\sigma_{G}, \sigma_{L}, \sigma_{O}\right)$ be a solution of Eqs. (3.2)-(3.4) on $(0, R)$, with boundary conditions (3.1) and no-flux conditions at $r=0$. From Proposition 3..2 we immediately infer that for $R \rightarrow \infty$ necessarily $\sigma_{G}(0) \rightarrow 0$, otherwise the r.h.s. of (3.2) would stay away from zero and it would be impossible to satisfy $\sigma_{G}(R)=\sigma_{G}^{*}$ for growing $R$.

Applying to $\sigma_{O}$ the same argument used for $\sigma_{G}$, we realise that either $\sigma_{L}(0)$ has to tend to zero or $\sigma_{O}(0) \rightarrow 0$. In both cases $f_{O}$ will tend to zero in $r=0$. Putting these pieces of information together and recalling the expression (2.6) for $f_{A T P}$ we conclude that, for $R$ exceeding a quantity dependent on the data, the value of $f_{A T P}$ in the center of the spheroid goes below the threshold $f_{N}$ and the thesis follows. By the same token we may actually assert that $f_{A T P}$ tends to zero uniformly as $R \rightarrow \infty$ in any given compact interval.

The above result asserts that sufficiently large spheroids cannot be fully viable. In what follows, we prove the existence of a necrotic interface, presenting at the same time a possible procedure for calculating its location. We proceed to a formal integration of the Cauchy problem for (3.2)-(3.4) with data (3.1) and

$$
\sigma_{i}^{\prime}(R)=\Sigma_{i}, \quad i=G, L, O,
$$

where $\Sigma_{G}>0$ and $\Sigma_{O}>0$. As we will see, the parameter $\Sigma_{G}$ is going to take a particular role. 
Performing two consecutive integrations we arrive at the system

$$
\begin{aligned}
& \sigma_{G}(r)=\sigma_{G}^{*}-R^{2} \Sigma_{G}\left(\frac{1}{r}-\frac{1}{R}\right)+\Gamma_{G} \int_{r}^{R}\left(\frac{1}{r}-\frac{1}{r^{\prime}}\right) r^{\prime 2} f_{G}\left(\sigma_{G}\left(r^{\prime}\right)\right) d r^{\prime} \\
& \sigma_{L}(r)=\sigma_{L}^{*}-R^{2} \Sigma_{L}\left(\frac{1}{r}-\frac{1}{R}\right)+\Gamma_{L} \int_{r}^{R}\left(\frac{1}{r}-\frac{1}{r^{\prime}}\right) r^{\prime 2} f_{L}\left(\sigma_{G}\left(r^{\prime}\right), \sigma_{L}\left(r^{\prime}\right), \sigma_{O}\left(r^{\prime}\right)\right) d r^{\prime} \\
& \sigma_{O}(r)=\sigma_{O}^{*}-R^{2} \Sigma_{O}\left(\frac{1}{r}-\frac{1}{R}\right)+\Gamma_{O} \int_{r}^{R}\left(\frac{1}{r}-\frac{1}{r^{\prime}}\right) r^{\prime 2} f_{O}\left(\sigma_{G}\left(r^{\prime}\right), \sigma_{L}\left(r^{\prime}\right), \sigma_{O}\left(r^{\prime}\right)\right) d r^{\prime} .
\end{aligned}
$$

We note that the equation for $\sigma_{G}$ can be solved autonomously. With the transformation $z=R-r$ (without modifying the symbols) it takes the form of a nonlinear Volterra equation

$$
\sigma_{G}(z)=\sigma_{G}^{*}-R \Sigma_{G} \frac{z}{R-z}+\Gamma_{G} \int_{0}^{z}\left(z-z^{\prime}\right) \frac{R-z^{\prime}}{R-z} f_{G}\left(\sigma_{G}\left(z^{\prime}\right)\right) d z^{\prime},
$$

uniquely solvable for $z<R$ (see Thm. 8.2.1 in [4], p.90). Likewise, we can obtain a system of nonlinear Volterra integral equations for $\sigma_{L}, \sigma_{O}$, for which the same existence and uniqueness result holds true (see Cor. 8.2.1 in [4], p.91).

Since we are looking for a necrotic region, in view of Proposition 3..1 we have to take $R$ larger than some $R_{N}$. For the chosen $R$, we also take $\Sigma_{G}<\Sigma_{G}^{\star}(R)$, where $\Sigma_{G}^{\star}(R)$ corresponds to the slope of the "natural" boundary value problem for (3.2) with conditions $\sigma_{G}^{\prime}(0)=0, \sigma_{G}(R)=\sigma_{G}^{*}$. Indeed, it will only be for $\Sigma_{G} \in\left(0, \Sigma_{G}^{\star}\right)$ that $\sigma_{G}(r)$ develops a positive minimum $\sigma_{G}(\rho)$ for some $\rho \in(0, R)$. The latter conclusion follows from

Lemma 3..1. Both $\sigma_{G}$ and $\sigma_{G}^{\prime}$ depend in a monotone way on $\Sigma_{G}$.

Proof. Differentiating the Volterra equation (3.10) w.r.t. $\Sigma_{G}$ we obtain

$$
\frac{\partial \sigma_{G}}{\partial \Sigma_{G}}=-R \frac{z}{R-z}+\Gamma_{G} \int_{0}^{z}\left(z-z^{\prime}\right) \frac{R-z^{\prime}}{R-z} \frac{d f_{G}}{d \sigma_{G}} \frac{\partial \sigma_{G}}{\partial \Sigma_{G}} d z^{\prime},
$$

from which we deduce

$$
\frac{\partial \sigma_{G}}{\partial \Sigma_{G}}<0
$$

Performing the same operation on

$$
r^{2} \sigma_{G}^{\prime}=R^{2} \Sigma_{G}-\Gamma_{G} \int_{r}^{R} r^{\prime 2} f_{G}\left(\sigma_{G}\right) d r^{\prime}
$$

we find

$$
r^{2} \frac{\partial \sigma_{G}^{\prime}}{\partial \Sigma_{G}}=R^{2}-\Gamma_{G} \int_{r}^{R} r^{\prime 2} \frac{d f_{G}}{d \sigma_{G}} \frac{\partial \sigma_{G}}{\partial \Sigma_{G}} d r^{\prime}
$$

and thanks to (3.11) we conclude

$$
\frac{\partial \sigma_{G}^{\prime}}{\partial \Sigma_{G}}>0
$$


In fact, inequalities (3.11) and (3.12) lead us to conclude the following

Proposition 3..3. For each $\Sigma_{G} \in\left(0, \Sigma_{G}^{\star}\right)$ there exists a function

$$
\rho=\rho\left(\Sigma_{G}\right),
$$

decreasing with $\Sigma_{G}$, that defines the point where the function $\sigma_{G}(r)$ takes its minimum. This minimum, in turn, decreases with $\Sigma_{G}$.

If we want $\rho$ to coincide with $\rho_{N}$, we must require that also $\sigma_{L}^{\prime}$ and $\sigma_{O}^{\prime}$ vanish at the same point. Since

$$
\begin{aligned}
& r^{2} \sigma_{L}^{\prime}=R^{2} \Sigma_{L}-\Gamma_{L} \int_{r}^{R} r^{\prime 2} f_{L}\left(\sigma_{G}, \sigma_{L}, \sigma_{O}\right) d r^{\prime}, \\
& r^{2} \sigma_{O}^{\prime}=R^{2} \Sigma_{O}-\Gamma_{O} \int_{r}^{R} r^{\prime 2} f_{O}\left(\sigma_{G}, \sigma_{L}, \sigma_{O}\right) d r^{\prime},
\end{aligned}
$$

we must impose the following conditions on $\Sigma_{G}, \Sigma_{L}, \Sigma_{O}$ :

$$
\begin{aligned}
\Sigma_{G} & =\frac{\Gamma_{G}}{R^{2}} \int_{\rho\left(\Sigma_{G}\right)}^{R} r^{2} f_{G}\left(\sigma_{G}\right) d r, \\
\Sigma_{L} & =\frac{\Gamma_{L}}{R^{2}} \int_{\rho\left(\Sigma_{G}\right)}^{R} r^{2} f_{L}\left(\sigma_{G}, \sigma_{L}, \sigma_{O}\right) d r, \\
\Sigma_{O} & =\frac{\Gamma_{O}}{R^{2}} \int_{\rho\left(\Sigma_{G}\right)}^{R} r^{2} f_{O}\left(\sigma_{G}, \sigma_{L}, \sigma_{O}\right) d r .
\end{aligned}
$$

The equations above simply express the mass balance and they can be obtained directly from (3.2)(3.4) by integration. Equation (3.13) is just the implicit definition of $\rho\left(\Sigma_{G}\right)$, and (3.14)-(3.15) can be interpreted in the form

$$
\begin{aligned}
& \Sigma_{L}-\Omega_{L}\left(\Sigma_{G}, \Sigma_{L}, \Sigma_{O}\right)=0, \\
& \Sigma_{O}-\Omega_{O}\left(\Sigma_{G}, \Sigma_{L}, \Sigma_{O}\right)=0,
\end{aligned}
$$

i.e. as the equation of a curve $\chi$ in the space $\left(\Sigma_{G}, \Sigma_{L}, \Sigma_{O}\right)$, parameterized by $\Sigma_{G}$. It is easily seen that the origin belongs to $\chi$. Of course $\chi$ exists for $\Sigma_{G}>0$ if the determinant

$$
J=\left|\begin{array}{cc}
1-\frac{\partial \Omega_{L}}{\partial \Sigma_{L}} & -\frac{\partial \Omega_{L}}{\partial \Sigma_{O}} \\
-\frac{\partial \Omega_{O}}{\partial \Sigma_{L}} & 1-\frac{\partial \Omega_{O}}{\partial \Sigma_{O}}
\end{array}\right|
$$

does not vanish. Moreover, we observe that the identity (2.5) implies

$$
\frac{1}{\Gamma_{O}} \Sigma_{O}-\frac{3}{\Gamma_{L}} \Sigma_{L}=\frac{6}{\Gamma_{G}} \Sigma_{G},
$$


which may replace for instance Eq. (3.16), with obvious computational advantage. Consequently, instead of $J$ we can compute

$$
\tilde{J}=\left|\begin{array}{cc}
\frac{3}{\Gamma_{L}} & -\frac{1}{\Gamma_{O}} \\
-\frac{\partial \Omega_{O}}{\partial \Sigma_{L}} & 1-\frac{\partial \Omega_{O}}{\partial \Sigma_{O}}
\end{array}\right|=\frac{3}{\Gamma_{L}}\left(1-\frac{\partial \Omega_{O}}{\partial \Sigma_{O}}\right)-\frac{1}{\Gamma_{O}} \frac{\partial \Omega_{O}}{\partial \Sigma_{L}},
$$

that contains only the derivatives of $\Omega_{O}$. In other words, we only need to study two of the four derivatives of $\Omega_{O}, \Omega_{L}$.

Thus we aim our analysis at the target of showing that $\tilde{J} \neq 0$, which will lead us to the result of main interest, namely

Theorem 3..1. For $R$ sufficiently large there exists one and only one solution $\sigma_{G}, \sigma_{L}, \sigma_{O}, \rho_{N}$ of the free boundary problem defined by Eqs. (3.2)-(3.4), (3.1), and (3.5)-(3.6).

Indeed, from the considerations above it will be enough to show that $\tilde{J} \neq 0$ (i.e. $J \neq 0$ ), since this will allow to continue the curve $\chi$ in the space $\left(\Sigma_{G}, \Sigma_{L}, \Sigma_{O}\right)$, evaluating the values of $\sigma_{G}, \sigma_{L}, \sigma_{O}$ at $r=\rho\left(\Sigma_{G}\right)$ to calculate the corresponding value of $f_{A T P}$. Moreover we know from Proposition 3..1 that, proceeding from $\Sigma_{G}=0$ to $\Sigma_{G}=\Sigma_{G}^{\star}$, the value of $f_{A T P}$ is bound to reach the threshold $f_{N}$ for some $\Sigma_{G}=\hat{\Sigma}_{G}$. The knowledge of $\hat{\Sigma}_{G}$ allows us to construct the solution of the free boundary problem and this procedure provides a unique solution. Therefore, the following Proposition is all we need to conclude that Theorem 3..1 holds true.

Proposition 3..4. The derivatives

$$
\frac{\partial \Omega_{O}}{\partial \Sigma_{L}}, \frac{\partial \Omega_{O}}{\partial \Sigma_{O}}
$$

are negative.

Proof. $\Omega_{O}$ is defined by the r.h.s. of (3.15). We have

$$
\begin{aligned}
& \frac{\partial \Omega_{O}}{\partial \Sigma_{L}}=\frac{\Gamma_{O}}{R^{2}} \int_{\rho\left(\Sigma_{G}\right)}^{R} r^{2}\left(\frac{\partial f_{O}}{\partial \sigma_{L}} \frac{\partial \sigma_{L}}{\partial \Sigma_{L}}+\frac{\partial f_{O}}{\partial \sigma_{O}} \frac{\partial \sigma_{O}}{\partial \Sigma_{L}}\right) d r \\
& \frac{\partial \Omega_{O}}{\partial \Sigma_{O}}=\frac{\Gamma_{O}}{R^{2}} \int_{\rho\left(\Sigma_{G}\right)}^{R} r^{2}\left(\frac{\partial f_{O}}{\partial \sigma_{L}} \frac{\partial \sigma_{L}}{\partial \Sigma_{O}}+\frac{\partial f_{O}}{\partial \sigma_{O}} \frac{\partial \sigma_{O}}{\partial \Sigma_{O}}\right) d r .
\end{aligned}
$$

From (2.12), we can say that

$$
\frac{\partial f_{O}}{\partial \sigma_{j}}>0, \quad j=L, O .
$$

Our aim is to prove that the integrands in (3.19), (3.20) are negative. 
To this end we reconsider equation (3.10) and we write the analogous equations for $\sigma_{L}, \sigma_{O}$ expressed by the variable $z=R-r$ :

$$
\begin{aligned}
& \sigma_{L}(z)=\sigma_{L}^{*}-R \Sigma_{L} \frac{z}{R-z}+\Gamma_{L} \int_{0}^{z}\left(z-z^{\prime}\right) \frac{R-z^{\prime}}{R-z} f_{L} d z^{\prime} \\
& \sigma_{O}(z)=\sigma_{O}^{*}-R \Sigma_{O} \frac{z}{R-z}+\Gamma_{O} \int_{0}^{z}\left(z-z^{\prime}\right) \frac{R-z^{\prime}}{R-z} f_{O} d z^{\prime}
\end{aligned}
$$

Then, using (2.5), we get an alternative expression for (3.22):

$$
\sigma_{L}(z)=\sigma_{L}^{*}-R \Sigma_{L} \frac{z}{R-z}+\Gamma_{L} \int_{0}^{z}\left(z-z^{\prime}\right) \frac{R-z^{\prime}}{R-z}\left(\frac{1}{3} f_{O}-2 f_{G}\right) d z^{\prime}
$$

We note that we are not concerned with the singularity of the kernel

$$
K\left(z, z^{\prime}\right)=\left(z-z^{\prime}\right) \frac{R-z^{\prime}}{R-z}>0
$$

as $z \rightarrow R$, i.e. $r \rightarrow 0$, because we will stop before reaching the center of the sphere (moreover, in case we do reach $r=0$ it can be shown that singular terms cancel out).

Now we perform the following derivatives

$$
\begin{aligned}
& \frac{\partial \sigma_{L}}{\partial \Sigma_{L}}=-R \frac{z}{R-z}+\frac{\Gamma_{L}}{3} \int_{0}^{z} K\left(z, z^{\prime}\right) \psi_{L}\left(z^{\prime}\right) d z^{\prime}, \\
& \frac{\partial \sigma_{O}}{\partial \Sigma_{L}}=\Gamma_{O} \int_{0}^{z} K\left(z, z^{\prime}\right) \psi_{L}\left(z^{\prime}\right) d z^{\prime}, \\
& \frac{\partial \sigma_{L}}{\partial \Sigma_{O}}=\frac{\Gamma_{L}}{3} \int_{0}^{z} K\left(z, z^{\prime}\right) \psi_{O}\left(z^{\prime}\right) d z^{\prime}, \\
& \frac{\partial \sigma_{O}}{\partial \Sigma_{O}}=-R \frac{z}{R-z}+\Gamma_{O} \int_{0}^{z} K\left(z, z^{\prime}\right) \psi_{O}\left(z^{\prime}\right) d z^{\prime},
\end{aligned}
$$

where

$$
\psi_{i}(z)=\frac{\partial f_{O}}{\partial \sigma_{L}} \frac{\partial \sigma_{L}}{\partial \Sigma_{i}}+\frac{\partial f_{O}}{\partial \sigma_{O}} \frac{\partial \sigma_{O}}{\partial \Sigma_{i}}, \quad i=L, O .
$$

By suitably combining (3.24)-(3.25) we can obtain the following Volterra integral equation for the function $\psi_{L}(z)$

$$
\psi_{L}(z)=-R \frac{z}{R-z} \frac{\partial f_{O}}{\partial \sigma_{L}}+\left(\frac{\Gamma_{L}}{3} \frac{\partial f_{O}}{\partial \sigma_{L}}+\Gamma_{O} \frac{\partial f_{O}}{\partial \sigma_{O}}\right) \int_{0}^{z} K\left(z, z^{\prime}\right) \psi_{L}\left(z^{\prime}\right) d z^{\prime},
$$

whose kernel is still positive, thanks to (3.21), and with the free term negative with a negative derivative for $z=0$. Thus we can easily conclude that

$$
\psi_{L}(z)<0 .
$$


Starting from (3.26)-(3.27), we can see in a completely similar way that

$$
\psi_{O}(z)<0 \text {. }
$$

Since (3.19), (3.20) are equivalent to

$$
\begin{aligned}
& \frac{\partial \Omega_{O}}{\partial \Sigma_{L}}=\frac{\Gamma_{O}}{R^{2}} \int_{\rho\left(\Sigma_{G}\right)}^{R} r^{2} \psi_{L} d r, \\
& \frac{\partial \Omega_{O}}{\partial \Sigma_{O}}=\frac{\Gamma_{O}}{R^{2}} \int_{\rho\left(\Sigma_{G}\right)}^{R} r^{2} \psi_{O} d r,
\end{aligned}
$$

the proof is concluded.

Remark 3..1. Thanks to identity (2.5), we can manipulate equations (3.8)-(3.9) so to decouple them. We can isolate the integral

$$
\int_{r}^{R}\left(\frac{1}{r}-\frac{1}{r^{\prime}}\right) r^{\prime 2} f_{O} d r^{\prime}
$$

in (3.9) and replace the obtained expression in (3.8). This leads to the equation

$\sigma_{L}=\sigma_{L}^{*}-R^{2} \Sigma_{L}\left(\frac{1}{r}-\frac{1}{R}\right)+\frac{\Gamma_{L}}{3 \Gamma_{O}}\left[\sigma_{O}-\sigma_{O}^{*}+R^{2} \Sigma_{O}\left(\frac{1}{r}-\frac{1}{R}\right)\right]-2 \Gamma_{L} \int_{r}^{R}\left(\frac{1}{r}-\frac{1}{r^{\prime}}\right) r^{\prime 2} f_{G} d r^{\prime}$.

If in addition we observe that

$$
\Gamma_{G} \int_{r}^{R}\left(\frac{1}{r}-\frac{1}{r^{\prime}}\right) r^{\prime 2} f_{G} d r^{\prime}=\sigma_{G}(r)-\sigma_{G}^{*}+R^{2} \Sigma_{G}\left(\frac{1}{r}-\frac{1}{R}\right),
$$

and we use this identity in (3.28), we obtain an explicit expression for $\sigma_{L}$ in terms of $\sigma_{O}$ and $\sigma_{G}$ :

$$
\begin{aligned}
\sigma_{L}= & \sigma_{L}^{*}-R^{2} \Sigma_{L}\left(\frac{1}{r}-\frac{1}{R}\right)+\frac{\Gamma_{L}}{3 \Gamma_{O}}\left[\sigma_{O}-\sigma_{O}^{*}+R^{2} \Sigma_{O}\left(\frac{1}{r}-\frac{1}{R}\right)\right] \\
& -\frac{2 \Gamma_{L}}{\Gamma_{G}}\left[\sigma_{G}-\sigma_{G}^{*}+R^{2} \Sigma_{G}\left(\frac{1}{r}-\frac{1}{R}\right)\right] .
\end{aligned}
$$

Placing now (3.29) back into (3.9), allows to eliminate $\sigma_{L}$.

We could use the equation (3.18) and the equation for $\sigma_{O}$ obtained by eliminating $\sigma_{L}$ between (3.8), (3.9) to reduce the determination of $\Sigma_{O}$ (and hence of $\Sigma_{L}$ ) as a function of $\Sigma_{G}$ to the solution of an equation involving only $\Sigma_{O}$ and $\sigma_{O}$, besides $\Sigma_{G}$ and $\sigma_{G}$. However such a procedure would turn out to be more complicated than dealing with the original system, which has the advantage of possessing some symmetry.

As a concluding comment, we note that the above existence proof remains valid even if the consumption rates are not precisely the ones proposed in section 2.2., but just possess the same qualitative properties. 


\section{Appendix}

Let us consider a cell at the steady state and assume that glucose, lactate and oxygen are the only metabolites exchanged with the extracellular space. Let $\phi_{G}, \phi_{P}, \phi_{L}^{+}$, and $\phi_{L}^{-}$denote the fluxes $\left(\mathrm{mol} \cdot \mathrm{cell}^{-1} \cdot \mathrm{s}^{-1}\right)$ glucose to pyruvate, pyruvate to Krebs cycle, pyruvate to lactate and, respectively, lactate to pyruvate (see Fig. 1).

Recalling (2.1)-(2.2), the net production rate of NADH in the reactions

$$
\text { Glucose } \rightarrow \text { Pyruvate } \rightleftharpoons \text { Lactate }
$$

is given by $2 \phi_{G}-\phi_{L}^{+}+\phi_{L}^{-}$, because when pyruvate is converted into lactate, 1 NADH molecule is consumed. Since each NADH (or $\mathrm{FADH}_{2}$ ) molecule requires $(1 / 2) \mathrm{O}_{2}$ to be oxidized, and the cumulative production rate of $\mathrm{NADH}$ and $\mathrm{FADH}_{2}$ from pyruvate oxidation is $5 \phi_{P}$, the oxygen consumption rate, $f_{O}$, is given by:

$$
f_{O}=\frac{5}{2} \phi_{P}+\phi_{G}-\frac{1}{2} \phi_{L}^{+}+\frac{1}{2} \phi_{L}^{-}
$$

Since in addition

$$
\phi_{P}=2 \phi_{G}-\phi_{L}^{+}+\phi_{L}^{-},
$$

it follows that

$$
f_{O}=3 \phi_{P}
$$

Moreover, taking into account that

$$
f_{G}=\phi_{G}, \quad f_{L}=\phi_{L}^{-}-\phi_{L}^{+}
$$

from (A.1) we obtain

$$
f_{O}=6 f_{G}+3 f_{L} .
$$

Denoting by $\eta_{1}$ and $\eta_{2}$ the mean number of ATP molecules generated as a consequence of the oxidation of $1 \mathrm{NADH}$ and $1 \mathrm{FADH}_{2}$ molecules, respectively, from reactions (2.1)-(2.3) we can write

$$
f_{A T P}=2 \phi_{G}+\phi_{P}+\eta_{1}\left(4 \phi_{P}+2 \phi_{G}-\phi_{L}^{+}+\phi_{L}^{-}\right)+\eta_{2} \phi_{P} .
$$

Note that the second term represents the production of GTP, considered equivalent to ATP. Since from (A.1) $\phi_{P}=f_{O} / 3$, the above equation can be rewritten as

$$
f_{A T P}=2 f_{G}+\left(5 \eta_{1}+\eta_{2}+1\right) f_{O} / 3 .
$$

\section{References}

[1] B. Alberts, D. Bray, J. Lewis, M. Raff, K. Roberts, J.D. Wastson. Molecular biology of the cell. Garland Publishing, New York, 1994, Chap. 11. 
[2] R.P. Araujo, D.L.S. McElwain. A history of the study of solid tumour growth: the contribution of mathematical modelling. Bull. Math. Biol., 66 (2004), 1039-1091.

[3] A.K. Bouzier, R. Goodwin, F. Macouillard-Poulletier de Gannes, H. Valeins, P. Voisin, P. Canioni, M. Merle. Compartmentation of lactate and glucose metabolism in C6 glioma cells. J. Biol. Chem., 273 (1998), 27162-27169.

[4] J.R. Cannon. The one-dimensional heat equation. In: Encyclopedia of Mathematics and its Applications, Vol. 23, Addison-Wesley Publishing Co., Menlo Park, CA., 1984.

[5] J.J. Casciari, S.V. Sotirchos, R.M. Sutherland. Variations in tumor cell growth rates and metabolism with oxygen concentration, glucose concentration, and extracellular $p H$. J. Cell. Physiol., 151 (1992), 386-394.

[6] J.J. Casciari, S.V. Sotirchos, R.M. Sutherland. Mathematical modelling of microenvironment and growth in EMT6/Ro multicellular tumour spheroids. Cell. Prolif., 25 (1992), 1-22.

[7] C. Dubois, A.E. Radunskaya. Cellular automaton model of melanoma growth incorporating metabolic dependencies on the microenvironment. Manuscript in preparation.

[8] G.L. Edlund, A.P. Halestrap. The kinetics of transport of lactate and pyruvate into rat hepatocytes. Biochem. J., 249 (1988), 117-126.

[9] J.P. Freyer, R.M. Sutherland. A reduction in the in situ rates of oxygen and glucose consumption of cells in EMT6/Ro spheroids during growth. J. Cell. Physiol., 124 (1985), 516-524.

[10] R.A. Gatenby, E.T. Gawlinski. The glycolytic phenotype in carcinogenesis and tumor invasion: insights through mathematical models. Cancer Res., 63 (2003), 3847-3854.

[11] M. Guppy, P. Leedman, X.L. Zu, V. Russel. Contribution by different fuels and metabolic pathways to the total ATP turnover of proliferating MCF-7 breast cancer cells. Biochem. J., 364 (2002), 309-315.

[12] P.C. Hinkle, M.A. Kumar, A. Resetar, D.L. Harris. Mechanistic stoichiometry of mitochondrial oxidative phosphorylation. Biochemistry, 30 (1991), 3576-3582.

[13] Y. Jiang, J. Pjesivac-Grbovic, C. Cantrell, J.P. Freyer. A multiscaale model for avascular tumor growth. Biophys. J., 89 (2005), 3884-3894.

[14] T. Schroeder, H. Yuan, B.L. Viglianti, C. Peltz, S. Asopa, Z. Vujaskovic, M.W. Dewhirst. Spatial heterogeneity and oxygen dependence of glucose consumption in R3230Ac and fibrosarcomas of the Fischer 344 rat. Cancer Res., 65 (2005), 5163-5171.

[15] K. Smallbone, D.J. Gavaghan, R.A. Gatenby, P.K. Maini. The role of acidity in solid tumour growth and invasion. J. Theor. Biol., 235 (2005), 476-484.

[16] L. Stryer. Biochemistry. W.H. Freeman and Company, New York, 1988, Chaps. 15-17. 
[17] R.M. Sutherland. Cell and environment interactions in tumor microregions: the multicell spheroid model. Science, 240 (1988), 177-184.

[18] R. Venkatasubramanian, M.A. Henson, N.S. Forbes. Incorporating energy metabolism into a growth model of multicellular tumor spheroids. J. Theor. Biol., 242 (2006), 440-453.

[19] O. Warburg. On the origin of cancer cells. Science, 123 (1956), 309-314. 